\title{
The Impact of Pharmacist Interventions on Reducing Medication Errors in Pediatric Patients: A Systematic Review and Meta-analysis
}

\author{
Lina Naseralallah¹, Tarteel Hussain'1, Myriam Jaam², Shane Pawluk² \\ 'Student, College of Pharmacy, QU Health, Qatar University \\ ${ }^{2}$ Faculty, College of Pharmacy, QU Health, Qatar University
}

\section{Background}

- Medication errors (MEs) are events that can occur at any stage of the medication use process including prescribing, transcription, dispensing, administration and monitoring ${ }^{1}$

- Preventable adverse drug reactions (ADRs) are medication-related injuries that arises as a result of an error ${ }^{1}$

- MEs and preventable ADRs are avoidable events that can result in significant patient harm ${ }^{1}$

- Clinical pharmacists play an integral role in preventing MEs and preventable ADRs in adults ${ }^{2}$

- Pediatric patients are more prone to MEs which have three times the potential to cause harm as compared to adults ${ }^{3}$

\section{Objectives}

- To qualitatively and quantitatively assess the effectiveness of pharmacist interventions on reducing medication errors for pediatric patients in hospital settings

To explore types of MEs that prompted pharmacist interventions in hospitalized pediatric patients

\section{Methods}

\section{Table 1. Study Methodology}

Study Design

Systematic review and metaanalysis following the PRISMA checklist

Protocol

Registration

Search Engines

ROSPERO registration number: CRD42019126541

Search Terms

Eligibility Criteria

PubMed, Embase, Cochrane, Google Scholar

Search categories:

Population:

"pediatrics"[MeSH]

- Intervention:

"pharmacists"[MeSH]

- Outcome: "medication errors"[MeSH]

\section{Inclusion:}

- Hospital settings

- Pediatrics (birth-18 years)

- Medication error rate

Exclusion:

- Intervention not solely made by pharmacist

Selection and Data Extraction

wo reviewers (independently)

\section{Author(s)}

Year of publication

Country of the study

- Study design

- Study site(s)

Population characteristics

Description of pharmacis

intervention

- Medication error rate

\section{Review Manager}

- Forest plot

- Random effect model

- Odds ratio

- Heterogeneity $\left(I^{2}\right)$

Crowe Critical Appraisal

Tool (CCAT)

Quality

Assessment

\section{Results}

- Out of 606 citations, 19 were included in

the qualitative synthesis and 6 in the

meta-analysis (Figure 1)

- Studies design:

Retrospective or prospective cohort studies $(\mathrm{n}=11)$

Before-after studies $(n=6)$

Cross-sectional studies $(n=2)$

- Departments included:

- Various departments within the hospital $(\mathrm{n}=8)$

Neonatal intensive care unit $(n=3)$

General medical ward $(n=3)$

Pediatric intensive care unit $(n=2)$

Surgery department $(n=1)$

Unspecified $(n=2)$

- Clinical pharmacist intervention:

Educational sessions $(n=5)$

Review or order validation $(n=5)$

- Various unit-based activities $(n=4)$

- Multiple pharmacist interventions $(n=3)$

Attending rounds $(n=2)$
Figure 1. PRISMA flow diagram of the study selection process

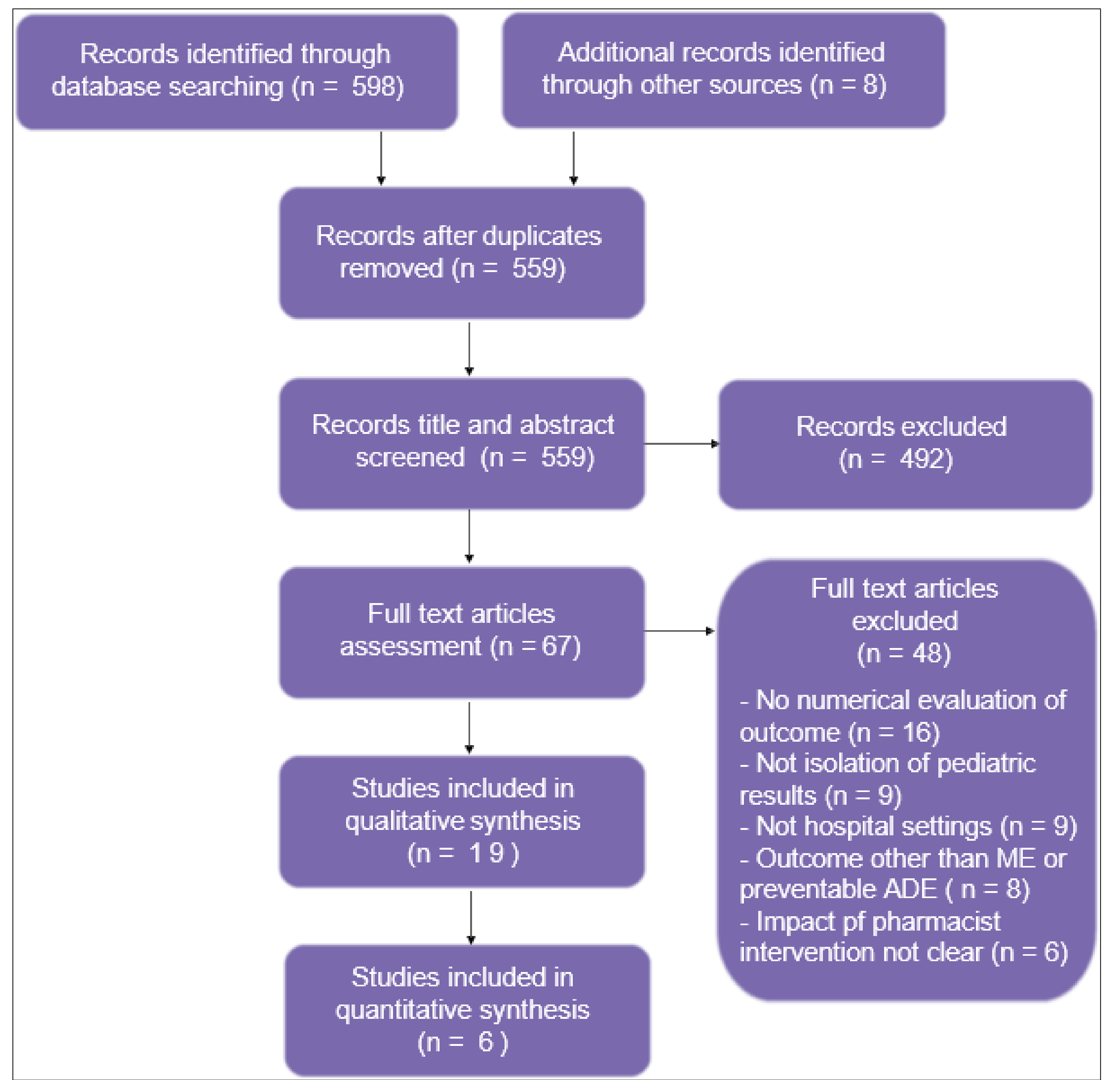

- Main types of MEs provoking clinical pharmacist interventions:
Wrong dose
Wrong frequency
Drug interaction
Wrong drug
Wrong formulation
Wrong administration rate

- Quality assessment: the overall quality of included studies is moderate (27.9/40) with high similarity between raters (ICC range, 0.948 to 0.997 )

- Meta-analysis:

The pooled odds ratio across 6 studies was 0.27 (95\% $\mathrm{Cl} 0.15$ to 0.49$)$, favouring the pharmacist intervention Results of these studies are substantially heterogeneous (Figure 2)

Figure 2. Forest plot of clinical pharmacist (RPh) effect on medication errors

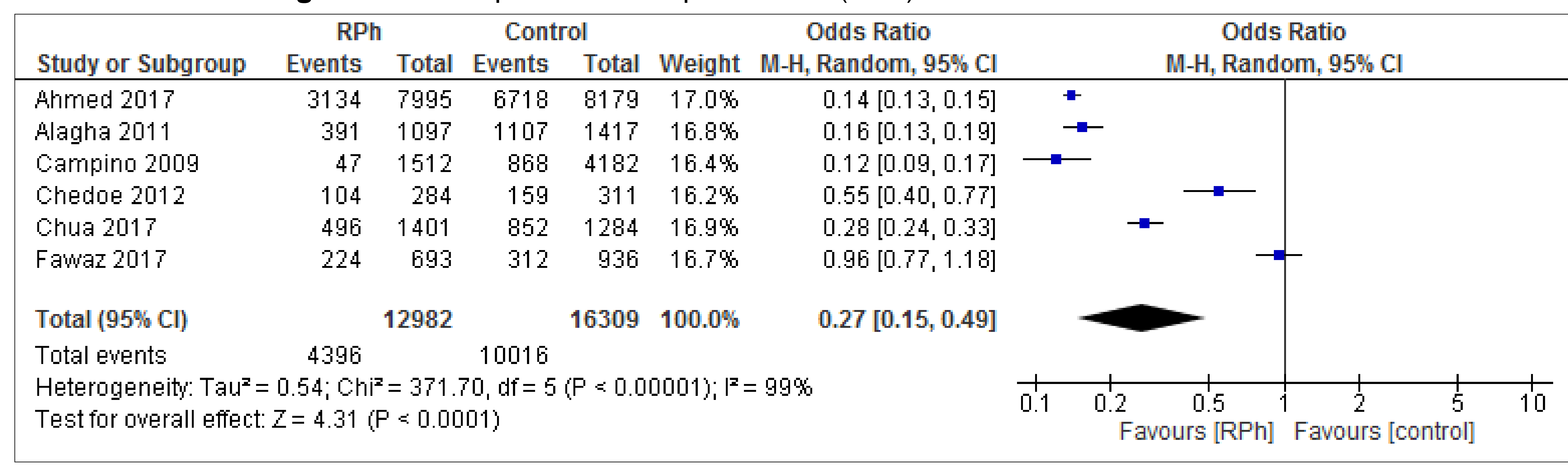

\section{Limitations}

- The overall quality of included studies is considered low to moderate

There is a high heterogeneity amongst studies as a result of the variations in settings (departments within the hospital) and types of pharmacist intervention (e.g. educational sessions, attending rounds, etc.)

\section{Future studies}

Conduct a subgroup analysis on the outcomes of the current study to evaluate the impact of pharmacist on particular MEs (e.g. administration errors)

- Assess the impact of clinical pharmacist interventions in primary healthcare clinics and community pharmacy settings

- Evaluate clinical pharmacist impact on other health outcomes (e.g. mortality, length of stay, emergency department visit) related to MEs

\section{Conclusions}

- Dosing errors are the most common type of ME in pediatric patients

- Pharmacist involvement through different types of interventions decreases the rate of ME occurrence, as compared to no pharmacist interventions for pediatric patients admitted to hospita

\section{References}

Morimoto T. Adverse drug events and medication errors: detection and classification methods. Quality and Safety in Health Care. 2004;13(4):306-314.

2. Wang $\mathrm{T}$, Benedict $\mathrm{N}$, Olsen $\mathrm{K}$ et al. Effect of critical care pharmacist's intervention on medication errors: A systematic review and metaanalysis of observational studies. Journal of Critical Care 2015;30(5):1101-1106

3. Costello JL, Torowicz DL, Yeh TS. Effects of a pharmacist-led American journal of health-system pharmacy : AJHP : official journal of pediatrics medication safety team on medication-error reporting. 